OPEN ACCESS

Edited by:

Rachel L. Roper,

The Brody School of Medicine at East Carolina University, United States

Reviewed by: Subramanian Dhandayuthapani, Texas Tech University Health Sciences Center, United States Brian Weinrick,

Trudeau Institute, United States

*Correspondence:

Chien-Fu Hung

chung2@jhmi.edu

${ }^{\dagger}$ Present Address: Yu-Min Chuang,

Section of Infectious Diseases, Department of Internal Medicine, Yale University School of Medicine, New

Haven, CT, United States

Received: 03 October 2017 Accepted: 23 January 2018 Published: 08 February 2018

Citation: Chuang Y-M, Pinn ML, Karakousis PC and Hung C-F (2018) Intranasa Immunization with DnaK Protein Induces Protective Mucosal Immunity against Tuberculosis in CD4-Depleted

Front. Cell. Infect. Microbiol. 8:31. doi: 10.3389/fcimb.2018.00031

\section{Intranasal Immunization with DnaK Protein Induces Protective Mucosal Immunity against Tuberculosis in CD4-Depleted Mice}

\author{
Yu-Min Chuang ${ }^{1 \dagger}$, Michael L. Pinn ${ }^{2}$, Petros C. Karakousis ${ }^{2,3}$ and Chien-Fu Hung ${ }^{1 *}$ \\ ${ }^{1}$ Department of Pathology, Johns Hopkins University School of Medicine, Baltimore, MD, United States, ${ }^{2}$ Department of \\ Medicine, Johns Hopkins University School of Medicine, Baltimore, MD, United States, ${ }^{3}$ Department of International Health, \\ Johns Hopkins Bloomberg School of Public Health, Baltimore, MD, United States
}

Mycobacterium tuberculosis (Mtb) remains a global health challenge due to the limited efficacy of the Mtb vaccine in current use, Bacillus Calmette-Guérin (BCG). To date, there is no available vaccine for immunocompromised individuals. Thus, there is an urgent need to develop a new vaccine candidate which can induce mucosal immunity in hosts with different immune statuses. DnaK (HSP70) has been shown to induce protective immunity against Mtb infection when administered by DNA vaccine; however, the protection is inferior to that induced by the BCG vaccine. In our study, we vaccinated C57BL/6J mice with DnaK protein alone. Subcutaneous or intranasal vaccination with DnaK generated IFN $\gamma$-secreting $\mathrm{CD}^{+}{ }^{+} \mathrm{T}$ cells in the spleen, but only intranasal vaccination generated IL-17-releasing CD4 ${ }^{+} T$ cells in the lungs, even when circulating CD4 ${ }^{+} \mathrm{T}$ cells were diminished. Furthermore, intranasal vaccination with DnaK generated tissue resident $C D 4^{+} T$ cells in the lungs. Vaccination with DnaK alone resulted in protective immunity comparable to BCG vaccination against tuberculosis in mice. Our results demonstrate that intranasal vaccination with DnaK can generate mucosal immunity in immunocompromised or immunocompetent mice and DnaK vaccination can generate protection against Mtb similar to BCG, underscoring its potential utility as an Mtb vaccine candidate in humans.

Keywords: tuberculosis, intranasal, DnaK, vaccine, immunodeficiency

\section{INTRODUCTION}

Tuberculosis (TB) is one of the primary causes of morbidity and mortality due to infectious diseases worldwide. Despite substantial research in the field, the Mycobacterium bovis Bacillus Calmette-Guérin (BCG) vaccine remains the only licensed preventive vaccine against $\mathrm{TB}$, which was introduced into clinical practice nearly a century ago. Although the BCG vaccine may protect children against some forms of TB, including central nervous system infections, its protective efficacy is highly variable and the vaccine does not confer protection against pulmonary TB in adults (Andersen and Doherty, 2005). The suboptimal efficacy of the BCG vaccine has been attributed to previous exposure to cross-reacting non-tuberculosis mycobacteria (Andersen and Doherty, 2005), chronic helminth infections (Elias et al., 2008), and the absence of key Mycobacterium tuberculosis (Mtb)-related antigens in the vaccine strain (Kalra et al., 2007). In addition, the live 
attenuated strain $M$. bovis BCG can escape host immunity and interfere with antigen presentation, contributing to reduced activation of T cells (Sendide et al., 2005; Russell et al., 2007; Pecora et al., 2009). The protective efficacy of the BCG vaccine is dependent on the induction of T-helper 1 (Th1) immune responses (Black et al., 2002), and recent studies suggest that boosting pre-exposure Th17 responses further enhances its protective efficacy (Chatterjee et al., 2011; Kleinnijenhuis et al., 2014). Multiple strategies have been used to improve the efficacy of the BCG vaccine, including genetic modification of the BCG strain (Bottai et al., 2015; Saiga et al., 2015), primeboost vaccination with a modified vaccinia virus Ankara (MVA) (Fletcher et al., 2016), and immunomodulatory techniques to enhance BCG immunogenicity (Jagannath et al., 2009; Speth et al., 2016). For example, toll-like receptor (TLR) agonists, which can activate innate immunity and promote antigen presentation (Lahiri et al., 2008), have shown promise as adjuvants in preclinical studies, although their immunological effects are variable (Gupta et al., 2016), and they may induce toxicity in humans (Steinhagen et al., 2011).

One possible explanation for the relatively modest protective efficacy of the BCG vaccine is that it is commonly administered via the subcutaneous route, which fails to induce significant mucosal immunity (Aguilo et al., 2014; Perdomo et al., 2016). On the other hand, mucosal vaccination with BCG generates IL-17-secreting antigen-specific $\mathrm{CD}^{+} \mathrm{T}$ cells in the mucosa and offers superior protection to challenge with virulent $\mathrm{Mtb}$ as compared to subcutaneous BCG vaccination (Aguilo et al., 2016; Perdomo et al., 2016). However, even if the efficacy of the BCG vaccine could be improved by use of alternative routes of administration, there are remaining concerns about the safety of using a live attenuated strain, since BCG vaccination has been associated with significant mortality and morbidity, particularly in children with primary immune deficiencies (Marciano et al., 2014) and those with AIDS (Hesseling et al., 2007), which are among the populations most susceptible to TB. Therefore, there is an urgent need to develop novel $\mathrm{TB}$ vaccination strategies to enhance vaccine potency among vulnerable immune-competent and immune-deficient populations.

Mycobacterial heat shock proteins serve as molecular chaperones for other proteins during stress conditions and help to recycle damaged proteins (Kim et al., 2013; Balchin et al., 2016). DnaK (HSP70) is a member of a small chaperone family, which is conserved in both prokaryotes and eukaryotes (Kim et al., 2013; Balchin et al., 2016). In Mtb, DnaK is encoded by an essential gene (Sassetti et al., 2003; Griffin et al., 2011), which is required for bacterial growth and proper protein folding (Fay and Glickman, 2014). DnaK can also activate innate immunity through TLR-2 and TLR-4 (Bulut et al., 2005; Tsan and Gao, 2009). Vaccination with DnaK linked innate and adaptive immunity and had an adjuvant effect during vaccination (Suzue and Young, 1996; Bulut et al., 2005). HSP70 fused with human papillomavirus (HPV) E6 as a DNA vaccine was found to generate $\mathrm{HPV}$-specific T-cell responses in patients (Trimble et al., 2009), and mycobacterial HSP70 has been shown to induce protective immunity by DNA vaccination in mice (Tascon et al., 1996; Lowrie et al., 1997). Furthermore, a DNA vaccine encoding HSP70, HSP65 and Apa given as a booster vaccine after BCG vaccination showed enhanced protection in the murine $\mathrm{TB}$ model compared to BCG vaccination alone (Ferraz et al., 2004). However, the protective effect was variable and inferior to BCG when vaccination with single antigens was administered (Lowrie et al., 1997). In the current study, we demonstrated that vaccination with recombinant DnaK protein alone by the subcutaneous (SC) or intranasal (IN) route can confer protective immunity. Only intranasal vaccination with DnaK could generate mucosal-associated IL-17-secreting CD4 ${ }^{+}$ $\mathrm{T}$ cells, even in systemic $\mathrm{CD} 4^{+}$-depleted mice, offering a potential new vaccine strategy against $\mathrm{TB}$.

\section{MATERIALS AND METHODS DnaK Protein Preparation}

H37Rv DnaK cloned into pMCSG73 (DNASU, clone: MtCD00587183) was used for expression of recombinant DnaK protein. The resulting plasmids were used to transform $E$. coli BL21 (DE3) RP competent cells (Stratagene). The transformed bacteria were selected by ampicillin $(100 \mu \mathrm{g} / \mathrm{ml})$. The expression and purification of His-tagged protein were performed as previously described (Soong et al., 2013). Lipopolysaccharide (LPS) was removed by the Endotoxin Removal Kit (GenScript), which can effective reduce LPS levels to less $0.1 \mathrm{EU} / \mathrm{ml}$.

\section{Vaccination of Mice}

Female C57BL/6J mice (6-8 weeks old) received subcutaneous (SC) injection of DnaK protein $(100 \mu \mathrm{g})$ or intranasal (IN) injection of DnaK protein $(20 \mu \mathrm{g})$ following anesthesia with ketamine/xylazine intraperitoneal injection. Another dosage of vaccination was given 2 weeks after the first vaccination. All procedures were performed according to protocols approved by the Johns Hopkins University Institutional Animal Care and Use Committee. Two weeks after the last vaccination, the mice were euthanized. Peripheral blood was collected by cardiac puncture and splenocytes were collected by mashing the spleens through a $100-\mu \mathrm{m}$ cell strainer and following lysis of red blood cells by ACK lysing buffer (Quality Biological). For BCG vaccination, Mycobacterium bovis Karlson and Lessel (BCG Pasteur, ATCC) was grown in Middlebrook 7H9 broth (BD Difco) supplemented with $10 \%$ oleic acid-albumin-dextrose-catalase (OADC) (BD), $0.1 \%$ glycerol, and $0.05 \%$ Tween -80 at $37^{\circ} \mathrm{C}$ on a shaker. BCG $\left(10^{6}\right.$ bacilli) at mid-logarithmic phase in $100 \mu$ l PBS was subcutaneously injected into C57BL/6J mice (6-8 weeks old) prior to aerosol challenge with Mtb.

\section{In Vivo CD4+ T-Cell Depletion}

For $\mathrm{CD}^{+}{ }^{+}$depletion studies, $300 \mu \mathrm{g}$ of the anti-CD4 monoclonal antibody, GK1.5 (BioXcell), was injected intraperitoneally into mice once and then $100 \mu \mathrm{g}$ of antibody was injected 1 week later. To test whether intranasal DnaK vaccination could generate intrapulmonary antigen-specific $\mathrm{T}$ cells following peripheral $\mathrm{CD}^{+}{ }^{+}$T-cell depletion, $200 \mu \mathrm{g}$ of GK1.5 antibody was injected 1 day before the first vaccinationand then $100 \mu \mathrm{g}$ of antibody was injected once weekly for 3 weeks. 


\section{Enzyme-Linked Immunosorbent Assay (ELISA)}

Antigen-specific antibody responses were measured by ELISA, as described previously (Cheng et al., 2001), with minor modifications in coating and serum incubation. The 96-well microplate was coated with purified DnaK protein $(1 \mu \mathrm{g} / \mathrm{ml})$ overnight. After blocking, sera from vaccinated mice were diluted 1:100 with PBS, added to wells, and incubated at room temperature for $2 \mathrm{~h}$.

\section{Intracellular Cytokine Staining and Flow Cytometry Analysis}

To detect antigen-specific $\mathrm{CD}^{+}{ }^{+}$T-cell responses by IFN- $\gamma$ and IL-17 intracellular staining, splenocytes were stimulated individually with recombinant $\operatorname{DnaK}(10 \mu \mathrm{g} / \mathrm{ml})$ for $24 \mathrm{~h}$ at $37^{\circ} \mathrm{C}$ before addition of GolgiPlug (BD Pharmingen, San Diego, CA) overnight. After incubation, the splenocytes were washed once with FACScan buffer and then stained with PEconjugated monoclonal rat anti-mouse CD4 (Clone RM4-5, BD Pharmingen, San Diego, CA). Cells were permeabilized using the Cytofix/Cytoperm kit (BD Pharmingen, San Diego, CA). Intracellular IFN- $\gamma$ was stained with FITC-conjugated rat antimouse IFN- $\gamma$ (Clone XMG1.2, BD Pharmingen, San Diego, CA) and IL-17 was stained with APC-conjugated rat anti-mouse IL17 (Clone TC11-18H10.1, BioLegend). At necropsy, the lungs were perfused with $1 \mathrm{ml}$ normal saline by direct injection into the right ventricle of the heart. A section of the lung was used for cytometry analysis and the tissue samples were incubated in $37^{\circ} \mathrm{C}$ for $1 \mathrm{~h}$ with intermittent agitation in RPMI medium (Gibco) containing collagenase D (1 mg/ml, Sigma), DNase $(0.25 \mathrm{mg} / \mathrm{ml}$, Sigma) and hyaluronidase type V (1 mg/ml, Sigma). The cells were then filtered through a $70-\mu \mathrm{m}$ nylon filter mesh to remove undigested tissue fragments and washed with completed RPMI medium. To measure antigen-specific IL-17- or IFN- $\gamma$-releasing pulmonary $\mathrm{T}$ cells, the cells from each group were stimulated with DnaK protein $(10 \mu \mathrm{g} / \mathrm{ml})$ and intracellular cytokine staining was performed as described above. For analysis of pulmonary $\mathrm{T}$ cell surface markers, the $\mathrm{T}$ cells from the lungs were collected as described above. Then, the cells were stained with FITCconjugated rat anti-mouse CD69 (clone H1.2F3, eBioscience), $\mathrm{PE}$-conjugated rat anti-mouse CD4 and PerCP-conjugated rat anti-mouse CD44 (clone IM7, eBioscience). Flow cytometry was performed with a FACSCalibur flow cytometer, and the results were analyzed with FlowJo software.

\section{Aerosol Infection of Mice with M. tuberculosis}

Wild-type M. tuberculosis (Mtb) H37Rv was grown in supplemented Middlebrook 7H9 broth, as previous described (Chuang et al., 2015). The mice were aerosol-infected using a Glas-Col Inhalation Exposure System (Terre Haute, IN) calibrated to deliver $\sim 100$ bacilli of wild-type Mtb H37Rv in a biosafety level-3 animal facility (Chuang et al., 2013). The mice were euthanized 28 days after aerosol challenge and the lungs were homogenized and plated for colony-formin units (CFU)
(Chuang et al., 2013) to evaluate the protective efficacy of the DnaK vaccination.

\section{Statistical Analysis}

Group means were compared by one-way analysis of variance analysis (ANOVA) with Tukey-Kramer post-hoc analysis, using MedCalc for Windows, version 16.2.1 (MedCalc Software, Ostend, Belgium). Data from at least three biological replicates were used to calculate means and standard error of the mean (SEM) for graphing purposes. To compare differences between experimental and control groups, statistical analysis employed the unpaired Student's $t$-test, and a $p<0.05$ was considered statistically significant.

\section{RESULTS}

\section{Vaccination of Mice with Recombinant DnaK Generates Antigen-Specific IFN $\gamma$-Secreting T Cells in the Spleens}

Immunological responses were measured 2 weeks after SC or IN vaccination of C57BL/6J mice with recombinant DnaK (Figure 1A). Both routes of vaccine administration generated detectable IgG responses by ELISA, although SC vaccination generated significantly higher levels of IgG response compared to IN vaccination (Figure 1B). Intracellular cytokine staining revealed that DnaK vaccination by the SC or IN routes generated a significant increase in IFN $\gamma$-secreting $\mathrm{CD} 4^{+} \mathrm{T}$ cells in the spleen after stimulation with recombinant protein but there was no significant difference between the SC and IN groups (Figure 1C). However, only IN vaccination generated a significant increase in antigen-specific IL-17-secreting $\mathrm{CD} 4^{+}$ splenocytes compared to the naïve group (Figure 1D, $p<0.05$ ). The vaccination study was repeated by using a different batch of recombinant DnaK protein and the results were similar (data not shown).

\section{Intranasal Vaccination with DnaK Generates Antigen-Specific IL-17-Secreting CD4 ${ }^{+}$T Cells in the Lungs}

Lymphocytes from the lung parenchyma were harvested from DnaK-vaccinated mice and stimulated with recombinant DnaK protein $(10 \mu \mathrm{g} / \mathrm{ml})$ for $36 \mathrm{~h}$. IN vaccination increased the proportion of $\mathrm{CD} 9^{+} \mathrm{CD} 4^{+} \mathrm{T}$ cells in the lungs without antigen stimulation (Figure 2A, $p<0.05$ ). Furthermore, intracellular cytokine staining revealed that IN vaccination generated significantly greater numbers of IL-17-secreting $\mathrm{CD} 4^{+} \mathrm{T}$ cells in the lungs compared to SC vaccination (Figure 2B, $p<0.05$ ). Here we have shown that IN vaccination with DnaK generated mucosal-associated immunity.

\section{Intranasal Vaccination with DnaK Generates Tissue-Resident Th17 T Cells in the Lungs}

To determine whether IN DnaK vaccination could generate lung tissue-resident $\mathrm{T}$ cells, mice vaccinated with recombinant DnaK by the IN route were treated with $\mathrm{CD} 4^{+}$-neutralizing antibodies 


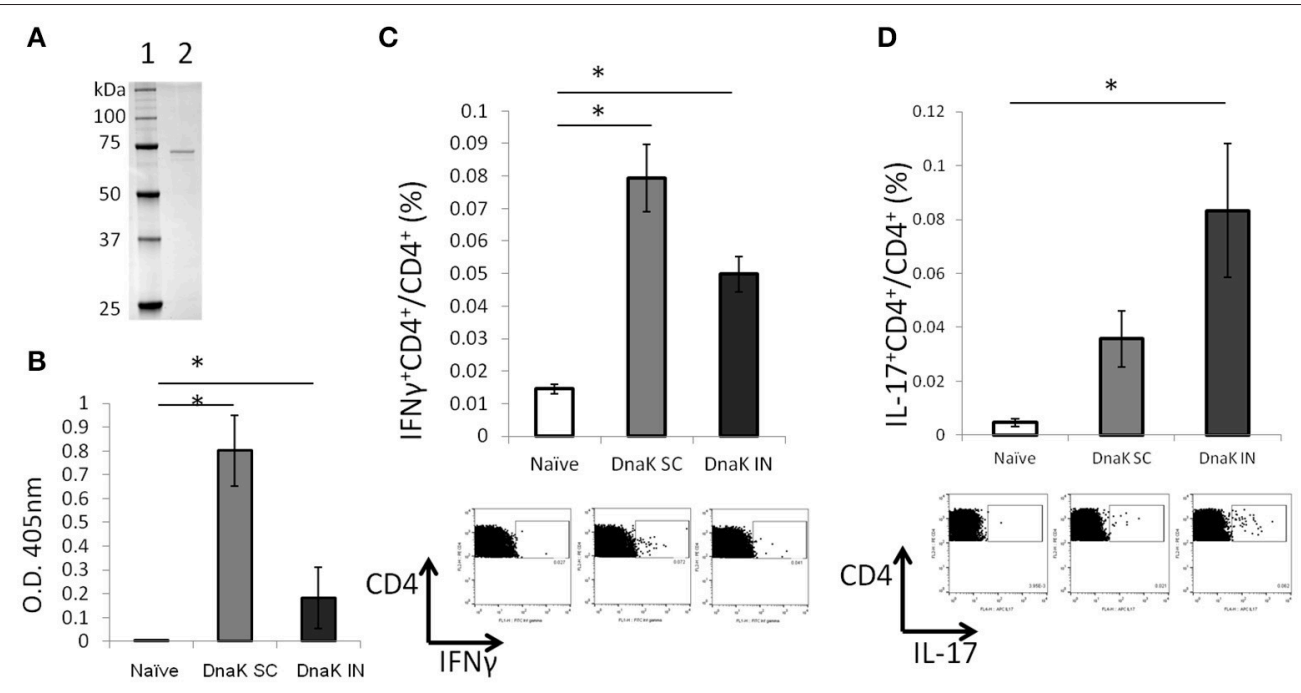

FIGURE 1 | Vaccination with DnaK protein alone generates immunoglobulin responses and antigen-specific CD4+ T cells. (A) SDS/PAGE analysis of purified DnaK protein. Lane 1: protein ladder, Lane 2: Purified His-tag Dnak protein. C57BL/6J mice were vaccinated twice with DnaK (100 $\mu$ g) by subcutaneous (SC) injection or with DnaK $(20 \mu \mathrm{g})$ by intranasal $(\mathrm{IN})$ injection. Immunological reponses were measured 2 weeks after the last vaccination. (B) Serum IgG responses to DnaK were measured by ELISA. Mean \pm SEM, $N=3-4,{ }^{*} p<0.05$ compared to the other two groups. (C,D) Splenocytes were stimulated with DnaK protein (10 $\left.\mu \mathrm{g} / \mathrm{ml}\right)$ and intracellular cytokine staining was used to detect antigen-specific CD4 ${ }^{+}$T cells secreting IFN $\gamma$ (B) and IL-17 (C). Mean \pm SEM, $N=3-4,{ }^{*} p<0.05$ compared to the other group.
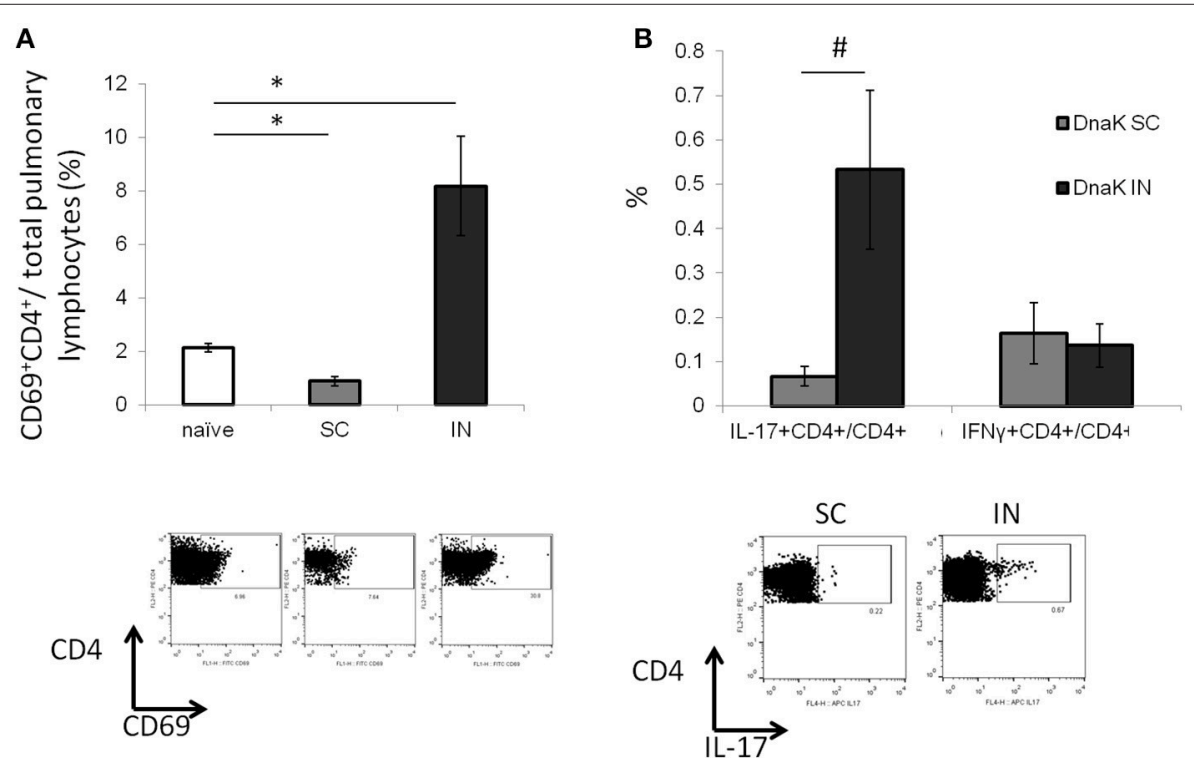

FIGURE 2 | Intranasal vaccination with recombinant DnaK protein generates IL-17-secreting CD4 ${ }^{+} \mathrm{T}$ cells in the lungs. C57BL/6J mice were vaccinated twice with DnaK $(100 \mu \mathrm{g})$ by subcutaneous injection or DnaK $(20 \mu \mathrm{g})$ by intranasal injection. Parenchymal intrapulmonary T cells were analyzed 2 weeks after the last vaccine dose. (A) After tissue digestion, unstimulated intrapulmonary CD69+ CD4 ${ }^{+}$T cells were measured by flow cytometry. $N=3-4,{ }^{*} p<0.05$ compared to compared to the other two groups. (B) Intrapulmonary T cells were stimulated with recombinant DnaK (10 $\mu \mathrm{g} / \mathrm{ml})$ and antigen-specific CD4 ${ }^{+} \mathrm{T}$ cells were evaluated for IFN $\gamma$ and IL-17 secretion by intracellular cytokine staining. Mean $\pm \mathrm{SEM}, N=3-4$, \#p $<0.05$ compared to SC-vaccinated mice.

after booster vaccination (Figure 3A). CD4 ${ }^{+}$T-cell depletion was confirmed by measuring total $\mathrm{CD}^{+}$T-cell numbers by flow cytometry 1 week after treatment with anti-CD $4^{+}$-neutralizing antibodies. There was $96.7 \pm 3 \%$ reduction of $\mathrm{CD}^{+} \mathrm{T}$ cells in spleens following $\mathrm{CD}^{+}{ }^{+} \mathrm{T}$-cell depletion compared to the control group. Antigen-specific IL-17-secreting $\mathrm{CD}^{+} \mathrm{T}$ cells were significantly reduced in the spleen but not in the lungs following CD4 ${ }^{+}$T-cell depletion (Figures 3B,D). Without $\mathrm{CD} 4{ }^{+}$ T-cell depletion, IN DnaK vaccination significantly increased the number of $\mathrm{CD} 44^{+} \mathrm{CD} 69^{+} \mathrm{CD} 4^{+} \mathrm{T}$ cells (Figure 3C), which have been identified as lung tissue-resident $\mathrm{T}$ cells (Zens et al., 2016). Similarly, during systemic $\mathrm{CD}^{+}$T-cell depletion, there 

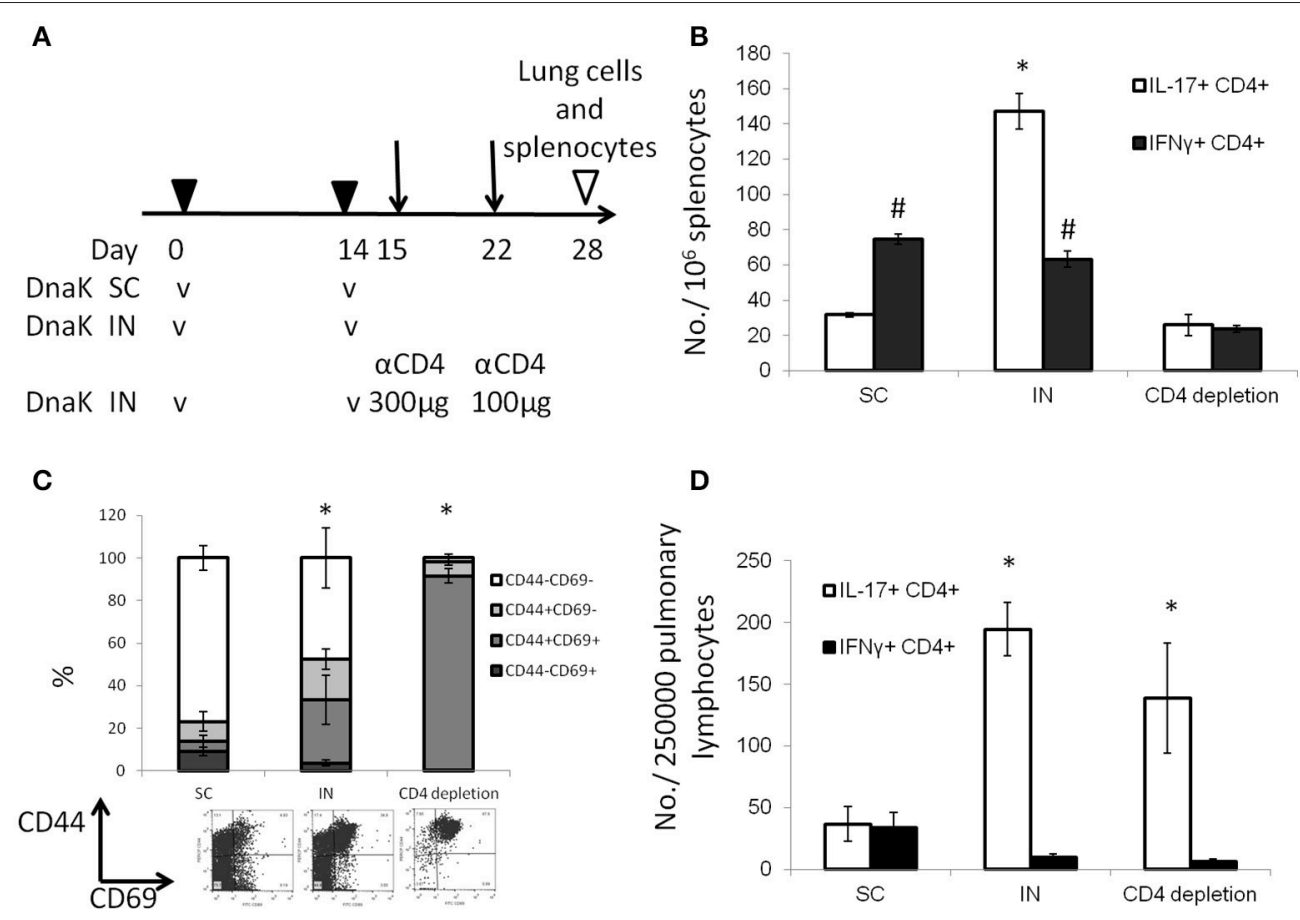

FIGURE 3 | Intranasal vaccination with DnaK generates tissue-resident T cells. C57BL/6J mice were vaccinated twice two weeks apart with DnaK (100 $\mu \mathrm{g}$ ) by subcutaneous injection or DnaK $(20 \mu \mathrm{g})$ by intranasal injection. One group of intranasally vaccinated mice received intraperitoneal injection of anti-CD4 ${ }^{+}$neutralizing antibody $(300 \mu \mathrm{g}) 1$ day after vaccination and another dose $(100 \mu \mathrm{g}) 1$ week later. Parenchymal T cells were analyzed 2 weeks after the last vaccine dose.

(A) Experiment scheme. (B) Splenocytes were stimulated with DnaK protein $(10 \mu \mathrm{g} / \mathrm{ml})$ and antigen-specific CD4+ T cells were assessed for IFN $\gamma$ and IL-17 secretion by intracellular cytokine staining. Mean $\pm \mathrm{SEM}, N=3-4,{ }^{*} p<0.05$ compared to the other two groups, $\# p<0.05$ compared to CD4 ${ }^{+}$depletion mice. (C) After tissue digestion, unstimulated intrapulmonary $\mathrm{CD} 69^{+} \mathrm{CD} 44^{+} \mathrm{CD} 4{ }^{+}$T cells were measured by flow cytometry. Mean $\pm \mathrm{SEM}, N=3-4,{ }^{*} p<0.05$ compared to the other two groups. (D) Intrapulmonary T cells were stimulated with recombinant DnaK $(10 \mu \mathrm{g} / \mathrm{ml})$ and antigen-specific CD4+ T cells were evaluated for IFN $\gamma$ and IL-17 secretion by intracellular cytokine staining. Mean $\pm \mathrm{SEM}, N=3-4,{ }^{*} p<0.05$ compared to compared to the other two groups.

was a notable increase in the proportion of $\mathrm{CD} 44^{+} \mathrm{CD} 69^{+} \mathrm{CD} 4^{+}$ $\mathrm{T}$ cells in the lungs of IN DnaK-vaccinated mice. Based these findings, IN vaccination with DnaK generates lung-resident IL17-secreting antigen-specific $\mathrm{CD} 4^{+} \mathrm{T}$ cells, which were resistant to systemic $\mathrm{CD} 4^{+}$depletion.

\section{Intranasal Vaccination with DnaK Generates Th17 T Cells in the Lungs during CD4 ${ }^{+}$T-Cell Depletion}

During chronic HIV infection, $\mathrm{CD} 4^{+} \mathrm{T}$ cells counts are gradually reduced to $<25 \%$ of the original numbers (Fauci et al., 1996). To determine if IN DnaK vaccination can generate immunity during $\mathrm{CD}^{+}$deficiency, control mice were vaccinated with DnaK $(20 \mu \mathrm{g})$ by IN injection or DnaK $(100 \mu \mathrm{g})$ by SC injection on Day 0 and Day 14. Experimental groups of mice received one of the vaccination schemes above, as well as intraperitoneal injection of anti-CD4 ${ }^{+}$neutralizing antibody one day before and during vaccination. Two weeks after the last vaccine dose, there was $65 \pm 4 \%$ reduction of splenic $\mathrm{CD}^{+} \mathrm{T}$ cells in mice receiving anti- $\mathrm{CD} 4^{+}$neutralizing antibodies compared to those that did not receive neutralizing antibodies. Antigenspecific $\mathrm{CD} 4^{+} \mathrm{T}$ cells were measured in the spleens and lungs. Intracellular cytokine staining revealed a significant reduction in antigen-specific IL-17-secreting $\mathrm{CD}^{+}$splenocytes in the $\mathrm{CD} 4^{+}$-depleted mice receiving IN or SC vaccination (Figure 4A). However, IN vaccination with DnaK still generated significant numbers of antigen-specific IL-17-secreting $\mathrm{CD} 4^{+}$cells in the lungs, even in $\mathrm{CD}_{4}^{+}$-depleted mice (Figure 4B).

\section{DnaK Vaccination Offers Protective Immunity Equivalent to BCG Vaccination}

C57BL/6J mice were vaccinated with DnaK $(100 \mu \mathrm{g})$ by SC or IN injection. Another group of mice were vaccinated with $10^{6}$ BCG Pasteur via SC injection. Eight weeks after vaccination, the mice were challenged with wild-type Mtb $\mathrm{H} 37 \mathrm{Rv}$ via the aerosol route. After 28 days of infection, the bacterial burden in the lungs was significantly lower in BCG- and DnaK-vaccinated groups $(p>0.05)$ compared to that in the unvaccinated group (Figure 5A) and there was no difference between BCG- and DnaK-vaccinated groups. To determine whether the protective effect of IN DnaK vaccination was dependent on the timing of vaccination relative to aerosol challenge and/or the specific DnaK protein preparation, C57BL/6J mice were vaccinated with an independently prepared $\mathrm{Mtb}$ recombinant $\mathrm{DnaK}$ protein via the IN route once a week for 3 weeks, either 90 days or 56 days before aerosol challenge (Figure 5B). Control mice were 


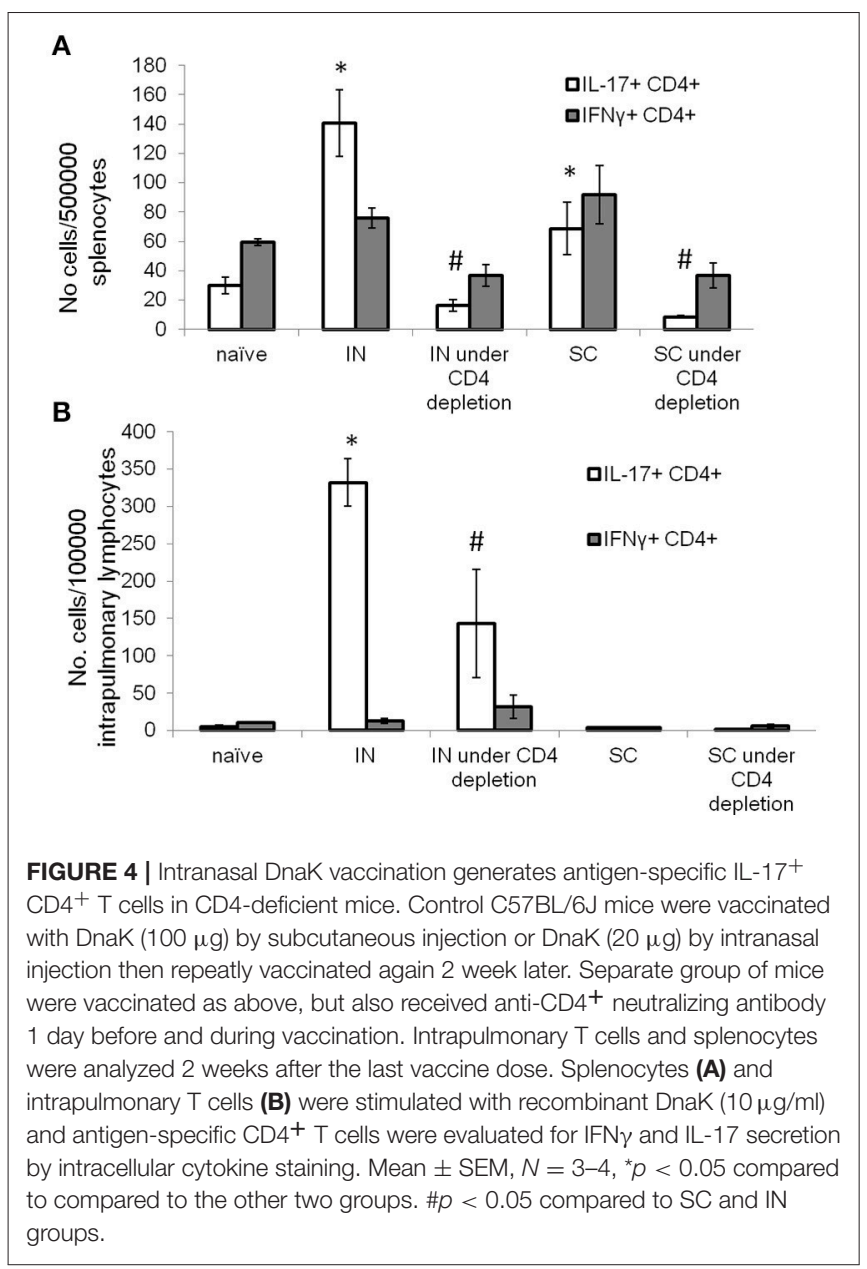

vaccinated via the SC route with $10^{6}$ BCG Pasteur strain, as described above, 56 days before aerosol challenge. All mice were aerosol-infected together and lung bacterial burden was assessed 30 days after infection. IN vaccination with DnaK provided consistent protection against Mtb aerosol challenge, including in the group that was vaccinated 3 months before challenge $(p<$ 0.05 compared to unvaccinated group).

\section{DISCUSSION}

Previous studies have shown that DNA vaccination targeting mycobacterial DnaK (HSP70) protects mice against challenge with Mtb, although this protection was inferior to that afforded by BCG (Lowrie et al., 1997). Limited data are available directly comparing the protective efficacy of protein- and DNA-based $\mathrm{TB}$ vaccines and different vaccination routes may yield distinct immunological responses (Britton and Palendira, 2003). BCG vaccination by the IN route shows superior protective efficacy compared to the SC route (Aguilo et al., 2016; Perdomo et al., 2016), perhaps due to the induction of increased numbers of tissue-resident IL-17-secreting $\mathrm{CD}^{+}{ }^{+} \mathrm{T}$ cells (Perdomo et al., 2016). Additional studies have also shown that vaccine presentation to the airways enhances control of Mtb infection in the lungs of mice (Derrick et al., 2014; Dai et al., 2016). In the current study, we show that IN vaccination with DnaK protein offers similar protection against Mtb challenge as SC BCG vaccination in mice, confirming the importance of the vaccine route of administration. This simple and effective vaccination strategy offers a new approach to protect against Mtb infection.

Following IN DnaK vaccination, the proportion of $\mathrm{CD} 44^{+} \mathrm{CD} 69^{+} \mathrm{CD}^{+} \mathrm{T}$ cells was significantly expanded. In the $\mathrm{CD} 4^{+}$T-cell-deficient mice, the majority of intrapulmonary $\mathrm{CD} 4^{+} \mathrm{T}$ cells following IN vacination were $\mathrm{CD} 44^{+} \mathrm{CD} 69^{+} \mathrm{CD} 4^{+}$ $\mathrm{T}$ cells, which are a marker of tissue resident memory $\mathrm{T}$ cells (Zens et al., 2016). At the same time, there was a similar number of intrapulmonary $\mathrm{IL}^{-17^{+}}$-secreting $\mathrm{CD} 4^{+} \mathrm{T}$ cells following $\mathrm{CD}^{+}$depletion. We showed that IN vaccination of DnaK alone can generate antigen-specific tissue-resident $\mathrm{T}$ cells in the lungs, which are maintained during systemic $\mathrm{CD} 4^{+} \mathrm{T}$-cell depletion. Tissue-resident $\mathrm{T}$ cells offer protective immunity against herpes simplex virus 2 (Shin and Iwasaki, 2012; Iijima and Iwasaki, 2014), influenza (Zens et al., 2016), and Mtb (Perdomo et al., 2016). Mucosal vaccination with BCG generates lung resident $\mathrm{T}$ cells, which provide enhanced protection compared to SC BCG vaccination (Perdomo et al., 2016). Therefore, mucosal vaccination appears to be a promising strategy to protect against $\mathrm{TB}$ infection. Here we show that IN vaccination with the recombinant protein DnaK generates lung tissue-resident $\mathrm{T}$ cells and offers equivalent protective immunity as BCG against TB, with fewer theoretical risks associated with a live attenuated vaccine, especially in the immunocompromised host.

TLR2 is required for production of IL-17 by mucosal CD4 ${ }^{+}$ $\mathrm{T}$ cells following IN vaccination and protection against different airway pathogens (Moffitt et al., 2014, 2015). In addition, TLR4 enhances IL-17 responses following mucosal vaccination with nanoemulsion adjuvants (Bielinska et al., 2010). DnaK can activate innate immunity through TLR2 and TLR4 (Bulut et al., 2005; Tsan and Gao, 2009), perhaps explaining its adjuvant properties during vaccination (Suzue and Young, 1996; Bulut et al., 2005), and offering a unique advantage as a TB preventive vaccine when administered via the IN route. In our study, we used the enodtoxin removal kit, which is effective in removing potential LPS contamination from purified DnaK protein. While we cannot entirely exclude the possibility of LPS-induced effects on T-cell responses following DnaK vaccination, we believe these effects are minimal for the following reasons. When using LPS as adjuvant during $\mathrm{SC}$ vaccination, there were significant $\mathrm{CD} 4^{+}$ T cells with mixed secretion of IFN $\gamma$, IL-4, or IL-17 (Kim et al., 2009; O'donnell et al., 2014; Caucheteux et al., 2017). In contrast, in our study, IFN $\gamma$-secreting $\mathrm{CD}^{+}{ }^{+} \mathrm{T}$ cells predominated in DnaK-vaccinated mice. In addition, the dose of LPS required to serve as adjuvant is greater than what could possibly remain after our protein purification. IN DnaK vaccination elicited antigenspecific $\mathrm{IL}-17^{+}$-secreting $\mathrm{CD} 4^{+} \mathrm{T}$ cells in the lungs, leading to similar protection as $\mathrm{SC}$-administered BCG vaccination.

During HIV infection, there is significant reduction of Th17-secreting $\mathrm{CD}^{+} \mathrm{T}$ cells in the gastrointestinal tract, although $\mathrm{Th}_{1}{ }^{+} \mathrm{CD}^{+} \mathrm{T}$ cells are relative preserved in broncheoalveolar lavage (Brenchley et al., 2008). Therefore, 
A
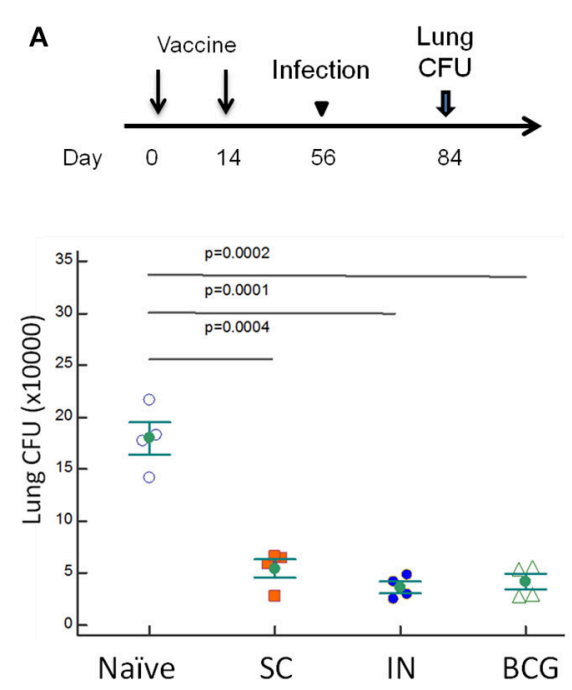
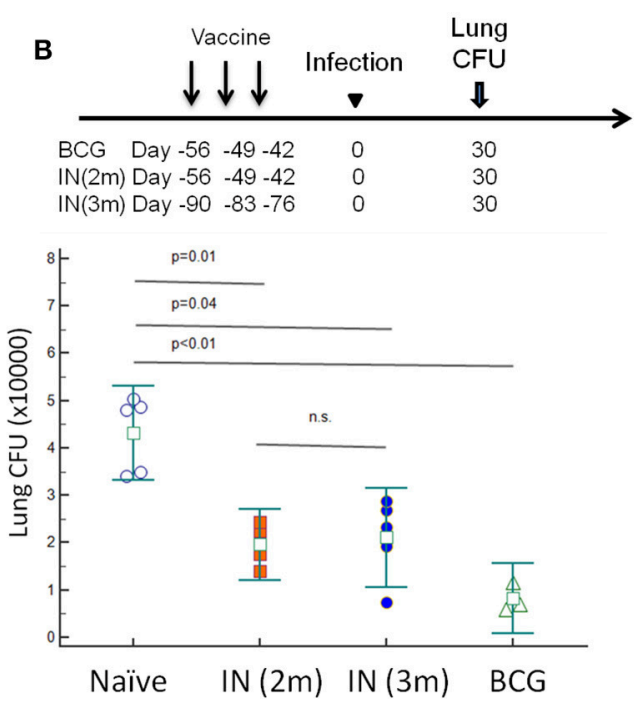

FIGURE 5 | Vaccination with DnaK protein confers equivalent protective immunity as BCG vaccination. (A) C57BL/6J mice were vaccinated twice with DnaK (100 $\mu \mathrm{g}$ ) by subcutaneous injection or DnaK $(20 \mu \mathrm{g})$ by intranasal injection. Control mice were subcutaneously vaccinated with BCG Pasteur once. Eight weeks after vaccination, all mice were aerosol-challenged with virulent $M$. tuberculosis $\mathrm{H} 37 \mathrm{Rv}$. Four weeks after infection, the lung bacillary burden (log 10 CFU) was determined. Mean \pm SEM, ${ }^{*} p<0.05$ (B). To confirm the protection of intranasal vaccination, C57BL/6J mice were vaccinated with DnaK (20 $\left.\mu \mathrm{g}\right)$ intranasally once a week for a total of 3 weeks, at two (IN $2 \mathrm{~m}$ ) or 3 months (IN $3 \mathrm{~m}$ ) before H37Rv aerosal infection in another experiment. The vaccinated and unvaccinated group of mice were infected at the same time. Lung bacillary burden $\left(\log _{10} \mathrm{CFU}\right)$ was determined 30 days after aerosol challenge. Mean $\pm \mathrm{SEM},{ }^{*} p<0.05$.

vaccination strategies capable of inducing $\mathrm{Th} 17^{+} \mathrm{CD} 4^{+} \mathrm{T}$ cells may potentially benefit HIV-infected individuals. Previous work has shown that administration of an Mtb Ag85A adenovirus vaccine to the respiratory mucosa generated Ag85A-specific $\mathrm{CD}^{+}{ }^{-} \mathrm{T}$ cells in $\mathrm{CD}_{4}^{+}$-depleted mice (Jeyanathan et al., 2010). Luminal $\mathrm{CD}^{+}{ }^{+} \mathrm{T}$ cells can be sustained and renewed without peripheral T-cell recruitment (Jeyanathan et al., 2010). However, limited information exists regarding whether IN vaccination can generate mucosal antigen-specific $\mathrm{CD}^{+} \mathrm{T}$ cells when peripheral $\mathrm{CD}^{+} \mathrm{T}$ cells are depleted. Here we show that IN vaccination with recombinant DnaK generated significant numbers of tissue-resident $\mathrm{IL}-17^{+} \mathrm{CD} 4^{+} \mathrm{T}$ cells in the lungs, even in mice unable to recruit systemic $\mathrm{T}$ cells following peripheral $\mathrm{CD} 4^{+}$T-cell depletion. This novel finding offers hope for novel TB vaccination strategies in patients with HIV/AIDS and other forms of cellular immune deficiency. It should be noted, however, that the presence of tissue-resident $\mathrm{CD}^{+} \mathrm{T}$ cells may reflect incomplete depletion due to reduced penetration of neutralizing antibodies into the lungs. Furthermore, it is unknown whether DnaK vaccine-induced lung-resident $\mathrm{IL}-17^{+} \mathrm{CD}^{+} \mathrm{T}$ cells could persist during long-term $\mathrm{CD} 4$ deficiency, as in the setting of untreated AIDS. Further studies are needed to address these questions.

\section{REFERENCES}

Aguilo, N., Alvarez-Arguedas, S., Uranga, S., Marinova, D., Monzon, M., Badiola, J., et al. (2016). Pulmonary but not subcutaneous delivery of BCG vaccine
In summary, IN vaccination of mice with DnaK generated lung tissue-resident IL-17-secreting $\mathrm{CD}^{+}{ }^{+} \mathrm{T}$ cells, offering similar protective efficacy against TB as SC BCG vaccination. The generation of intrapulmonary IL-17-secreting $\mathrm{CD}^{+} \mathrm{T}$ cells following IN DnaK vaccination was observed even following depletion of circulating $\mathrm{CD} 4^{+} \mathrm{T}$ cells, offering a novel potential vaccination strategy in immunocompromised hosts. Further studies are required to confirm the protective efficacy in other animal models, including non-human primates, and in the context of other immune deficiencies.

\section{AUTHOR CONTRIBUTIONS}

Y-MC, PCK, and C-FH designed the experiments. Y-MC and MP performed experiments. Y-MC and C-FH performed analyses. PCK provided funding for the experiments. Y-MC, PCK, and $\mathrm{C}-\mathrm{FH}$ wrote the manuscript.

\section{FUNDING}

Research reported in this publication was supported by the National Institute of Allergy and Infectious Diseases of the National Institutes of Health under Award Numbers R21 AI122922 to PCK. 
BCG vaccination confers dose-dependent superior protection compared to that of subcutaneous vaccination. Clin. Vaccine Immunol. 21, 594-597. doi: 10.1128/CVI.00700-13

Andersen, P., and Doherty, T. M. (2005). The success and failure of BCG implications for a novel tuberculosis vaccine. Nat. Rev. Microbiol. 3, 656-662. doi: $10.1038 /$ nrmicro1211

Balchin, D., Hayer-Hartl, M., and Hartl, F. U. (2016). In vivo aspects of protein folding and quality control. Science 353:aac4354. doi: 10.1126/science.aac4354

Bielinska, A. U., Gerber, M., Blanco, L. P., Makidon, P. E., Janczak, K. W., Beer, M., et al. (2010). Induction of Th17 cellular immunity with a novel nanoemulsion adjuvant. Crit. Rev. Immunol. 30, 189-199. doi: 10.1615/CritRevImmunol.v30.i2.60

Black, G. F., Weir, R. E., Floyd, S., Bliss, L., Warndorff, D. K., Crampin, A. C., et al. (2002). BCG-induced increase in interferon-gamma response to mycobacterial antigens and efficacy of BCG vaccination in Malawi and the UK: two randomised controlled studies. Lancet 359, 1393-1401. doi: 10.1016/S0140-6736(02)08353-8

Bottai, D., Frigui, W., Clark, S., Rayner, E., Zelmer, A., Andreu, N., et al. (2015). Increased protective efficacy of recombinant BCG strains expressing virulenceneutral proteins of the ESX-1 secretion system. Vaccine 33, 2710-2718. doi: 10.1016/j.vaccine.2015.03.083

Brenchley, J. M., Paiardini, M., Knox, K. S., Asher, A. I., Cervasi, B., Asher, T. E., et al. (2008). Differential Th17 CD4 T-cell depletion in pathogenic and nonpathogenic lentiviral infections. Blood 112, 2826-2835. doi: 10.1182/blood-2008-05-159301

Britton, W. J., and Palendira, U. (2003). Improving vaccines against tuberculosis. Immunol. Cell Biol. 81, 34-45. doi: 10.1046/j.0818-9641.2002.01143.x

Bulut, Y., Michelsen, K. S., Hayrapetian, L., Naiki, Y., Spallek, R., Singh, M., et al. (2005). Mycobacterium tuberculosis heat shock proteins use diverse Toll-like receptor pathways to activate pro-inflammatory signals. J. Biol. Chem. 280, 20961-20967. doi: 10.1074/jbc.M411379200

Caucheteux, S. M., Hu-Li, J., Mohammed, R. N., Ager, A., and Paul, W. E. (2017). Cytokine regulation of lung Th17 response to airway immunization using LPS adjuvant. Mucosal Immunol. 10, 361-372. doi: 10.1038/mi.2016.54

Chatterjee, S., Dwivedi, V. P., Singh, Y., Siddiqui, I., Sharma, P., Van Kaer, L., et al. (2011). Early secreted antigen ESAT-6 of Mycobacterium tuberculosis promotes protective $\mathrm{T}$ helper 17 cell responses in a toll-like receptor-2-dependent manner. PLoS Pathog. 7:e1002378. doi: 10.1371/journal.ppat.1002378

Cheng, W. F., Hung, C. F., Hsu, K. F., Chai, C. Y., He, L., Ling, M., et al. (2001). Enhancement of sindbis virus self-replicating RNA vaccine potency by targeting antigen to endosomal/lysosomal compartments. Hum. Gene Ther. 12, 235-252. doi: 10.1089/10430340150218387

Chuang, Y. M., Bandyopadhyay, N., Rifat, D., Rubin, H., Bader, J. S., and Karakousis, P. C. (2015). Deficiency of the novel exopolyphosphatase Rv1026/PPX2 leads to metabolic downshift and altered cell wall permeability in Mycobacterium tuberculosis. MBio 6:e02428. doi: 10.1128/mBio.02428-14

Chuang, Y. M., Belchis, D. A., and Karakousis, P. C. (2013). The polyphosphate kinase gene ppk2 is required for Mycobacterium tuberculosis inorganic polyphosphate regulation and virulence. MBio 4, e00039-e00013. doi: $10.1128 / \mathrm{mBio} .00039-13$

Dai, G., Rady, H. F., Huang, W., Shellito, J. E., Mason, C., and Ramsay, A. J. (2016). Gene-based neonatal immune priming potentiates a mucosal adenoviral vaccine encoding mycobacterial Ag85B. Vaccine 34, 6267-6275. doi: 10.1016/j.vaccine.2016.10.065

Derrick, S. C., Kolibab, K., Yang, A., and Morris, S. L. (2014). Intranasal administration of Mycobacterium bovis BCG induces superior protection against aerosol infection with Mycobacterium tuberculosis in mice. Clin. Vaccine Immunol. 21, 1443-1451. doi: 10.1128/CVI.00394-14

Elias, D., Britton, S., Aseffa, A., Engers, H., and Akuffo, H. (2008). Poor immunogenicity of BCG in helminth infected population is associated with increased in vitro TGF-beta production. Vaccine 26, 3897-3902. doi: 10.1016/j.vaccine.2008.04.083

Fauci, A. S., Pantaleo, G., Stanley, S., and Weissman, D. (1996). Immunopathogenic mechanisms of HIV infection. Ann. Intern. Med. 124, 654-663. doi: 10.7326/0003-4819-124-7-199604010-00006

Fay, A., and Glickman, M. S. (2014). An essential nonredundant role for mycobacterial DnaK in native protein folding. PLoS Genet. 10:e1004516. doi: 10.1371/journal.pgen.1004516
Ferraz, J. C., Stavropoulos, E., Yang, M., Coade, S., Espitia, C., Lowrie, D. B., et al. (2004). A heterologous DNA priming-Mycobacterium bovis BCG boosting immunization strategy using mycobacterial Hsp70, Hsp65, and Apa antigens improves protection against tuberculosis in mice. Infect. Immun. 72, 6945-6950. doi: 10.1128/IAI.72.12.6945-6950.2004

Fletcher, H. A., Snowden, M. A., Landry, B., Rida, W., Satti, I., Harris, S. A., et al. (2016). T-cell activation is an immune correlate of risk in BCG vaccinated infants. Nat. Commun. 7:11290. doi: 10.1038/ncomms11290

Griffin, J. E., Gawronski, J. D., Dejesus, M. A., Ioerger, T. R., Akerley, B. J., and Sassetti, C. M. (2011). High-resolution phenotypic profiling defines genes essential for mycobacterial growth and cholesterol catabolism. PLoS Pathog. 7:e1002251. doi: 10.1371/journal.ppat.1002251

Gupta, N., Vedi, S., Kunimoto, D. Y., Agrawal, B., and Kumar, R. (2016). Novel lipopeptides of ESAT-6 induce strong protective immunity against Mycobacterium tuberculosis: routes of immunization and TLR agonists critically impact vaccine's efficacy. Vaccine 34, 5677-5688. doi: 10.1016/j.vaccine.2016.08.075

Hesseling, A. C., Marais, B. J., Gie, R. P., Schaaf, H. S., Fine, P. E., Godfrey-Faussett, P., et al. (2007). The risk of disseminated Bacille Calmette-Guerin (BCG) disease in HIV-infected children. Vaccine 25, 14-18. doi: 10.1016/j.vaccine.2006.07.020

Iijima, N., and Iwasaki, A. (2014). T cell memory. a local macrophage chemokine network sustains protective tissue-resident memory CD4 T cells. Science 346, 93-98. doi: 10.1126/science. 1257530

Jagannath, C., Lindsey, D. R., Dhandayuthapani, S., Xu, Y., Hunter, R. L. Jr., and Eissa, N. T. (2009). Autophagy enhances the efficacy of BCG vaccine by increasing peptide presentation in mouse dendritic cells. Nat. Med. 15, 267-276. doi: $10.1038 / \mathrm{nm} .1928$

Jeyanathan, M., Mu, J., Mccormick, S., Damjanovic, D., Small, C. L., Shaler, C. R., et al. (2010). Murine airway luminal antituberculosis memory CD8 T cells by mucosal immunization are maintained via antigen-driven in situ proliferation, independent of peripheral T cell recruitment. Am. J. Respir. Crit. Care Med. 181, 862-872. doi: 10.1164/rccm.200910-1583OC

Kalra, M., Grover, A., Mehta, N., Singh, J., Kaur, J., Sable, S. B., et al. (2007). Supplementation with RD antigens enhances the protective efficacy of BCG in tuberculous mice. Clin. Immunol. 125, 173-183. doi: 10.1016/j.clim.2007.07.007

Kim, Y. E., Hipp, M. S., Bracher, A., Hayer-Hartl, M., and Hartl, F. U. (2013). Molecular chaperone functions in protein folding and proteostasis. Annu. Rev. Biochem. 82, 323-355. doi: 10.1146/annurev-biochem-060208-092442

Kim, Y. S., Hong, S. W., Choi, J. P., Shin, T. S., Moon, H. G., Choi, E. J., et al. (2009). Vascular endothelial growth factor is a key mediator in the development of $\mathrm{T}$ cell priming and its polarization to type 1 and type $17 \mathrm{~T}$ helper cells in the airways. J. Immunol. 183, 5113-5120. doi: 10.4049/jimmunol.0901566

Kleinnijenhuis, J., Quintin, J., Preijers, F., Benn, C. S., Joosten, L. A., Jacobs, C., et al. (2014). Long-lasting effects of BCG vaccination on both heterologous Th1/Th17 responses and innate trained immunity. J. Innate Immun. 6, 152-158. doi: 10.1159/000355628

Lahiri, A., Das, P., and Chakravortty, D. (2008). Engagement of TLR signaling as adjuvant: towards smarter vaccine and beyond. Vaccine 26, 6777-6783. doi: 10.1016/j.vaccine.2008.09.045

Lowrie, D. B., Silva, C. L., Colston, M. J., Ragno, S., and Tascon, R. E. (1997). Protection against tuberculosis by a plasmid DNA vaccine. Vaccine $15,834-838$. doi: 10.1016/S0264-410X(97)00073-X

Marciano, B. E., Huang, C. Y., Joshi, G., Rezaei, N., Carvalho, B. C., Allwood, Z., et al. (2014). BCG vaccination in patients with severe combined immunodeficiency: complications, risks, and vaccination policies. J. Allergy Clin. Immunol. 133, 1134-1141. doi: 10.1016/j.jaci.2014.02.028

Moffitt, K., Howard, A., Martin, S., Cheung, E., Herd, M., Basset, A., et al. (2015). T(H)17-Mediated protection against pneumococcal carriage by a whole-cell vaccine is dependent on toll-like receptor 2 and surface lipoproteins. Clin. Vaccine Immunol. 22, 909-916. doi: 10.1128/CVI.0 0118-15

Moffitt, K., Skoberne, M., Howard, A., Gavrilescu, L. C., Gierahn, T., Munzer, S., et al. (2014). Toll-like receptor 2-dependent protection against pneumococcal carriage by immunization with lipidated pneumococcal proteins. Infect. Immun. 82, 2079-2086. doi: 10.1128/IAI.01632-13

O'donnell, H., Pham, O. H., Li, L. X., Atif, S. M., Lee, S. J., Ravesloot, M. M., et al. (2014). Toll-like receptor and inflammasome signals converge to amplify 
the innate bactericidal capacity of T helper 1 cells. Immunity 40, 213-224. doi: 10.1016/j.immuni.2013.12.013

Pecora, N. D., Fulton, S. A., Reba, S. M., Drage, M. G., Simmons, D. P., Urankar-Nagy, N. J., et al. (2009). Mycobacterium bovis BCG decreases MHC-II expression in vivo on murine lung macrophages and dendritic cells during aerosol infection. Cell. Immunol. 254, 94-104. doi: 10.1016/j.cellimm.2008.07.002

Perdomo, C., Zedler, U., Kühl, A. A., Lozza, L., Saikali, P., Sander, L. E., et al. (2016). Mucosal BCG vaccination induces protective lungresident memory $\mathrm{T}$ cell populations against tuberculosis. MBio 7:e01686-16. doi: $10.1128 / \mathrm{mBio} .01686-16$

Russell, M. S., Iskandar, M., Mykytczuk, O. L., Nash, J. H., Krishnan, L., and Sad, S. (2007). A reduced antigen load in vivo, rather than weak inflammation, causes a substantial delay in $\mathrm{CD}^{+}{ }^{+}$T cell priming against Mycobacterium bovis (bacillus Calmette-Guerin). J. Immunol. 179, 211-220. doi: 10.4049/jimmunol.179.1.211

Saiga, H., Nieuwenhuizen, N., Gengenbacher, M., Koehler, A. B., Schuerer, S., Moura-Alves, P., et al. (2015). The recombinant BCG deltaurec::hly vaccine targets the AIM2 inflammasome to induce autophagy and inflammation. J. Infect. Dis. 211, 1831-1841. doi: 10.1093/infdis/jiu675

Sassetti, C. M., Boyd, D. H., and Rubin, E. J. (2003). Genes required for mycobacterial growth defined by high density mutagenesis. Mol. Microbiol. 48, 77-84. doi: 10.1046/j.1365-2958.2003.03425.x

Sendide, K., Deghmane, A. E., Pechkovsky, D., Av-Gay, Y., Talal, A., and Hmama, Z. (2005). Mycobacterium bovis BCG attenuates surface expression of mature class II molecules through IL-10-dependent inhibition of cathepsin S. J. Immunol. 175, 5324-5332. doi: 10.4049/jimmunol.175.8.5324

Shin, H., and Iwasaki, A. (2012). A vaccine strategy that protects against genital herpes by establishing local memory T cells. Nature 491, 463-467. doi: 10.1038/nature11522

Soong, R. S., Trieu, J., Lee, S. Y., He, L., Tsai, Y. C., Wu, T. C., et al. (2013). Xenogeneic human p53 DNA vaccination by electroporation breaks immune tolerance to control murine tumors expressing mouse p53. PLoS ONE 8:e56912. doi: 10.1371/journal.pone.0056912
Speth, M. T., Repnik, U., and Griffiths, G. (2016). Layer-by-layer nanocoating of live Bacille-Calmette-Guerin mycobacteria with poly(I:C) and chitosan enhances pro-inflammatory activation and bactericidal capacity in murine macrophages. Biomaterials 111, 1-12. doi: 10.1016/j.biomaterials.2016.09.027

Steinhagen, F., Kinjo, T., Bode, C., and Klinman, D. M. (2011). TLR-based immune adjuvants. Vaccine 29, 3341-3355. doi: 10.1016/j.vaccine.2010.08.002

Suzue, K., and Young, R. A. (1996). Adjuvant-free hsp70 fusion protein system elicits humoral and cellular immune responses to HIV-1 p24. J. Immunol. 156, 873-879.

Tascon, R. E., Colston, M. J., Ragno, S., Stavropoulos, E., Gregory, D., and Lowrie, D. B. (1996). Vaccination against tuberculosis by DNA injection. Nat. Med. 2, 888-892. doi: 10.1038/nm0896-888

Trimble, C. L., Peng, S., Kos, F., Gravitt, P., Viscidi, R., Sugar, E., et al. (2009). A phase I trial of a human papillomavirus DNA vaccine for HPV $16^{+}$cervical intraepithelial neoplasia 2/3. Clin. Cancer Res. 15, 361-367. doi: 10.1158/1078-0432.CCR-08-1725

Tsan, M. F., and Gao, B. (2009). Heat shock proteins and immune system. J. Leukoc. Biol. 85, 905-910. doi: 10.1189/jlb.0109005

Zens, K. D., Chen, J. K., and Farber, D. L. (2016). Vaccine-generated lung tissue-resident memory $\mathrm{T}$ cells provide heterosubtypic protection to influenza infection. JCI Insight 1:e85832. doi: 10.1172/jci.insight.85832

Conflict of Interest Statement: The authors declare that the research was conducted in the absence of any commercial or financial relationships that could be construed as a potential conflict of interest.

Copyright (c) 2018 Chuang, Pinn, Karakousis and Hung. This is an open-access article distributed under the terms of the Creative Commons Attribution License (CC $B Y)$. The use, distribution or reproduction in other forums is permitted, provided the original author(s) and the copyright owner are credited and that the original publication in this journal is cited, in accordance with accepted academic practice. No use, distribution or reproduction is permitted which does not comply with these terms. 